\title{
UN CAS DE PARASITISME ACCIDENTEL PAR UNE LARVE PRIMAIRE DE STREPSIPTĖRE CHEZ UN PHLÉBOTOME
}

\author{
Par J. RAGEAU
}

En étudiant un lot de Phlébotomes récoltés à Bafia (Ouest Cameroun), le 13 septembre 1949, nous avons observé chez un mâle de Phlebotomus schwetzi Adler, Theodor et Parrot, 1929, une larve primaire de Strepsiptère située à la partie inférieure du thorax de l'insecte, près du cou, et au-dessus des coxæ antérieures. Cette larve campodéiforme a $0,110 \mathrm{~mm}$. de long sur $0,06 \mathrm{~mm}$. de largeur maxima. La tête porte deux taches oculaires noires et deux longues soies ventrales dirigées vers l'arrière; les antennes et les pièces buccales sont vestigiales. Le thorax présente trois paires de pattes à larges coxæ, fémurs et tibias cylindriques, tarses longs et sétiformes. Abdomen à 10 segments, revètu de fortes soies raides sur les côtés; le dernier segment porte deux longues soies caudales et il existe deux appendices sétiformes sur la face ventrale du $8^{\circ}$ segment. Suivant la clé de R. M. Bohart (cité par Essig), cette larve appartiendrait à la famille des Halictophagidx.

Le Phlébotome hôte était en tous points semblable à un mâle normal et ne semblait pas affecté par son parasite. Celui-ci doit être très rare (1 sur 809 exemplaires de phlébotomes examinés) et sa localisation à l'intérieur du thorax d'un Diptère paraît anormale. Selon Nassonow (1909) et Ulrich (1927), les larves primaires de Strepsiptères, comme les triongulins des Coléoptères Meloidæ, ont une vie libre et s'accrochent aux insectes floricoles (notamment les Hyménoptères), pour se faire véhiculer jusqu'à leur hôte qui est généralement la larve du même insecte.

Dans le cas que nous avons observé, il s'agit d'une larve primaire de Strepsiptère qui s'est vraisemblablement égarée chez un Diptère. On sait que chez les Phlébotomes, les femelles seules sont hématophages, les mâles se nourrissant de jus sucrés. Le mâle de $P$. schwetzi a pu récolter son parasite en se posant sur une fleur envahie par des larves de Strepsiptère. Ceux-ci ne sont pas rares au

Ann. de Parasitologie, T. XXVI, $x^{\circ} 5-6 .-1951$. 
Cameroun à Yaoundé et Douala : nous avons récolté, en 1949, deux mâles de Strepsiptères, l'un sur une feuille de haricot, l'autre attiré par la lumière électrique, ainsi que plusieurs femelles parasitant l'abdomen de guêpes du genre Belonogaster à Yaoundé et M. le Professeur Roubaud en a cbservé sur des Vespida du genre Ropalidia (= Icaria) provenant de Douala et élevés à l'Institut Pasteur de Paris.

M. E. Séguy, sous-directeur du Laboratoire d'Entomologie au Muséum, a bien voulu nous confirmer que ce parasite appartenait à l'ordre des Strepsiptères et n’avait jamais été signalé chez les phlébotomes. Nous le prions d'agréer tous nos remerciements pour ses précieux conseils.

\section{RÉSUMÉ}

Dans cette note, nous signalons la présence, chez un mâle de Phlcbotomus schwetzi (Dipt. Psychodidæ), d'une larve primaire de Strepsiptère. Nous pensons qu'il s'agit d'un parasitisme accidentel.

\section{Bibliographie}

Adlen (S.), Theodon (O.) et Pannot (L.), - Rev. Zool. Bot. afr., XVIII, 1929, 73. Вонart (R. M.). - Pan. Pac. Ent., XII, 1936, 9-18 (cité par Essig).

- Ibid., XIII, 1937, 49-57 (cité par Essig).

Brumpt (E.). - Précis de Parasitologie, II, 1949, 1435, Masson, Paris.

Essig (E. O.). - College Entomology, 1942, 609-618, MacMillan, Ncw-York.

Inss (A. D.). - A general Textbook of Entomology, 1948, Mcthuen, London.

Nissonow. - Untersuchungen zur Naturgeschichte der Strepsipteren. Ber. Naturw. med. Ver., XXXIII, 1910, Innsbruck (cité par Imms).

Neveu-Lemarre (M.). - Trailé d'Entomologie médicale et vétérinaire, 1938, Vigot, Paris.

PIERCE (W. D.). - A monographic revision of the twisted winged insects comprising the order Strepsiptera Kirby, 1909. U.S. Nat. Mus. Bull., LXVI; $1-132$.

- The comparative morphology of the order Strepsiptera together with records and deseriptions of species. Proc. U.S. Nat. Mus., LIV, 1918, 319-501.

-- Strepsiptera. Genera Insectorum, XXI, 1911, 1-5̆4.

Ulnich (W.). - Strepsiptera. Biol. Tiere Deutschlands, XLI, 1927, 1-103.

Institut Pasteur de Paris

et Office de la Recherche scientifique d'Outre-Mer 